\title{
Draft genome sequence of Bosea sp. WAO an arsenite and sulfide oxidizer isolated from a pyrite rock outcrop in New Jersey
}

\author{
Alexandra B. Walczak ${ }^{1 *}$, Nathan Yee ${ }^{2}$ and Lily Y. Young ${ }^{2}$
}

\begin{abstract}
This genome report describes the draft genome and physiological characteristics of Bosea sp. WAO (=DSM 102914), a novel strain of the genus Bosea in the family Bradyrhizobiaceae. Bosea sp. WAO was isolated from pulverized pyritic shale containing elevated levels of arsenic. This aerobic, gram negative microorganism is capable of facultative chemolithoautotrophic growth under aerobic conditions by oxidizing the electron donors arsenite, elemental sulfur, thiosulfate, polysulfide, and amorphous sulfur. The draft genome is of a single circular chromosome 6,125,776 bp long consisting of 21 scaffolds with a $G+C$ content of $66.84 \%$. A total 5727 genes were predicted of which 5665 or $98.92 \%$ are protein-coding genes and 62 RNA genes. We identified the genes aio $A$ and $a i o B$, which encode the large and small subunits of the arsenic oxidase respectively. We also identified the genes for the complete sulfur oxidation pathway sox which is used to oxidize thiosulfate to sulfate.
\end{abstract}

Keywords: Neutrophilic sulfur oxidizer, Sox, Arsenite oxidase gene, Aio, Geomicrobiology, Microbe-mineral interactions, carbon fixation RuBisCO

\section{Introduction}

Bosea sp. WAO (white arsenic oxidizer) was enriched from a pulverized sample of weathered black shale obtained from an outcropping near Trenton, NJ that contained high levels of arsenic [1]. Bosea sp. WAO belongs to the class Alphaproteobacteria and family Bradyrhizobiaceae which currently consists of 12 genera: Bradyrhizobium, Afipia, Agromonas, Balneimonas, Blastobacter, Bosea, Nitrobacter, Oligotropha, Rhodoblastus, Rhodopseudomomonas, Salinarimonas, and Tardiphaga [2]. This phenotypically diverse family is composed of microorganisms that are involved in nitrogen cycling, human diseases, phototropism in non-sulfur environments, plant commensalism, and chemolithoautotrophic growth [2]. 16S rRNA gene analysis of the Bradyrhizobiaceae family indicates that

\footnotetext{
* Correspondence: Alexandra.Walczak@Rutgers.edu

${ }^{1}$ Division of Life Sciences, Rutgers University, The State University of New Jersey, Piscataway, New Jersey, USA

Full list of author information is available at the end of the article
}

the Bosea genus is most closely related to the genus Salinarimonas which currently consists of two species, Salinarimonas rosea and Salinarmonas ramus [2]. The microorganisms belonging to the genus Bosea have been isolated from a variety of environments such as soils, sediments, hospital water systems, and digester sludge [3-5]. The type strain Bosea thiooxidans $\mathrm{BI}-42^{\mathrm{T}}$ is capable of thiosulfate oxidation and the initial genus definition included this characteristic [3]. In 2003 La Scola emended the genus description to remove thiosulfate oxidation as a key descriptor after isolation of several other Bosea spp. that were unable to oxidized thiosulfate [4]. These organisms have a very diverse metabolism but their common characteristics include being Gramnegative, aerobic, rod shaped, motile, good growth between 25 to $35{ }^{\circ} \mathrm{C}$, intolerant to salt concentrations above $6 \% \mathrm{NaCl}$ and have been described to be heterotrophic [3-5]. Using selective enrichment and isolation techniques with arsenite $[\mathrm{As}(\mathrm{III})]$ as the sole 
electron donor Bosea sp. WAO was isolated under autotrophic conditions [1]. Here we summarize the physiological features together with the draft genome sequence and data analysis of Bosea sp. WAO.

\section{Organism information}

\section{Classification and features}

The genus Bosea has nine species with validly published names isolated from various environments: $B$. thiooxidans BI- $42^{\mathrm{T}}$ (AF508803) from agricultural soil [3], B. eneae $34614^{\mathrm{T}}$ (AF288300), B. vestrisii $34635^{\mathrm{T}}$ (AF288306), and B. massiliensis $63287^{\mathrm{T}}$ (AF288309) from a hospital water system [4], B. minatitlanensis AMX51 $^{\mathrm{T}}$ (AF273081) from anaerobic digester sludge [5] B. lupini R-45681 ${ }^{\mathrm{T}}$ (FR774992), B. lathyri R$46060^{\mathrm{T}}$ (FR774993), and $B$. robiniae $\mathrm{R}-46070^{\mathrm{T}}$ (FR774994) from the root nodules of legumes [6], and B. vaviloviae Vaf- $18^{\mathrm{T}}(\mathrm{KJ} 848741)$ from the root nodules of Vavilovia formosa [7]. Strain WAO's previously published identity was confirmed using the EzTaxon server [8]. The highest 16S rRNA pairwise similarities for strain WAO were found with the type strains $B$. vestrisii $34635^{\mathrm{T}}(99.72 \%)$, B. eneae $34614^{\mathrm{T}}(99.65 \%), \quad$ B. lupini R-45681 ${ }^{\mathrm{T}}(99.65 \%), \quad B$. thiooxidans $\mathrm{BI}-42^{\mathrm{T}}(99.24 \%)$, B. robiniae $\mathrm{R}-46070^{\mathrm{T}}$
(98.88\%), B. massiliensis $63287^{\mathrm{T}}$ (98.81\%), B. minatitlanensis $\mathrm{AMX51}^{\mathrm{T}}$ (98.48\%) and B. lathyri R$46060^{\mathrm{T}}$ (98.18\%). Phylogenetic analysis based on the $16 \mathrm{~S}$ rRNA gene of Bosea spp. and phylogenetically related organisms placed Bosea sp. WAO closest to the type strain B. lupini DSM $26673^{\mathrm{T}}$ with $B$. vestrisii $34635^{\mathrm{T}}$ and $B$. eneae $34614^{\mathrm{T}}$ in the same cluster (Fig. 1, Table 1). An average nucleotide identity analysis (ANI) score between strain WAO and B. lupini DSM $26673^{\mathrm{T}}$ of $84.64 \%$ was computed using IMG/ER [9]. This value is lower than the ANI species demarcation threshold range (95-96\%) [10]. To further identify Bosea sp. WAO to the species level phylogenic trees based on the housekeeping genes $a t p D, d n a K, \quad r e c A, \operatorname{gyr} B$ and $r p o B$ were produced from available Bosea and related Bradyrhizobiaceae type strains using MEGA7 (Figs. 2, 3, 4, 5, 6 and 7). Strain WAO did not consistently group with any of the type strains for all five genes further suggesting that it is a separate species. The ability of $B$. lupini to oxidize thiosulfate has not been determined [6]; however, B. vestrisii, B. eneae, and B. massiliensis have been determined to not oxidize thiosulfate to sulfate [4]. These results suggest that strain WAO represents a distinct species in the genus Bosea.

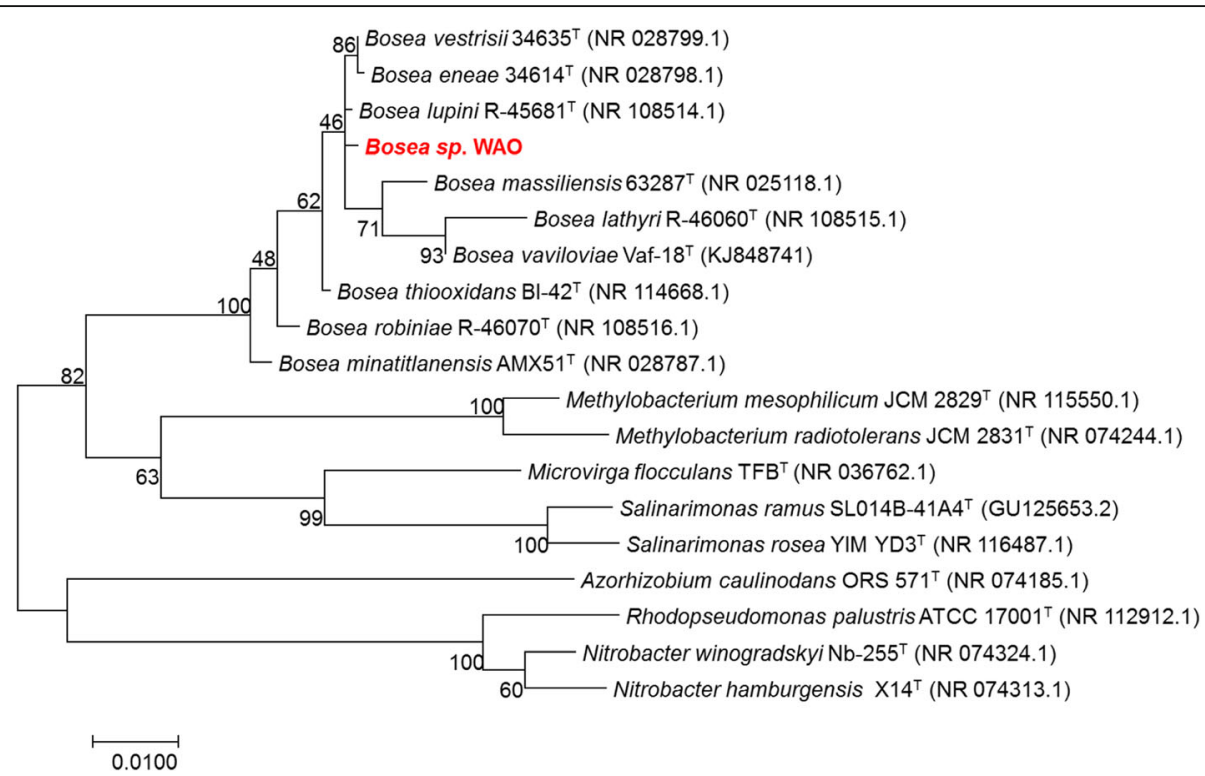

Fig. 1 Molecular Phylogenetic analysis by Maximum Likelihood method of the 16S rRNA gene. A Phylogenetic tree highlighting the position of Bosea sp. WAO relative to the other Bosea spp. based on the $16 \mathrm{~s}$ rRNA gene. The evolutionary history was inferred by using the Maximum Likelihood method based on the Tamura-Nei model [19]. The tree with the highest log likelihood $(-4792.5378)$ is shown. The percentage of trees in which the associated taxa clustered together is shown next to the branches. Initial tree(s) for the heuristic search were obtained automatically by applying Neighbor-Join and BioNJ algorithms to a matrix of pairwise distances estimated using the Maximum Composite Likelihood (MCL) approach, and then selecting the topology with superior log likelihood value. The tree is drawn to scale, with branch lengths measured in the number of substitutions per site. The analysis involved 19 nucleotide sequences. All positions containing gaps and missing data were eliminated. There were a total of 1376 positions in the final dataset. Evolutionary analyses were conducted in MEGA7 [20]. Type strains are indicated with a superscript T 
Table 1 Classification and general features of Bosea sp. WAO [22]

\begin{tabular}{|c|c|c|c|}
\hline MIGS ID & Property & Term & Evidence code ${ }^{a}$ \\
\hline & Classification & Domain Bacteria & $\operatorname{TAS}[23,24]$ \\
\hline & & Phylum Proteobacteria & TAS [25] \\
\hline & & Class Alphaproteobacteria & $\operatorname{TAS}[26,27]$ \\
\hline & & Order Rhizobiales & $\operatorname{TAS}[27,28]$ \\
\hline & & Family Bradyrhizobiaceae & $\operatorname{TAS}[27,29]$ \\
\hline & & Genus Bosea & $\operatorname{TAS}[3,30]$ \\
\hline & & Species Bosea sp. & TAS [24] \\
\hline & & Strain: WAO (DSM 102914) & TAS [1] \\
\hline & Gram stain & Negative & IDA \\
\hline & Cell shape & Rod & TAS [1] \\
\hline & Motility & Motile & TAS [1] \\
\hline & Sporulation & Not reported & NAS \\
\hline & Temperature range & Mesophile & IDA \\
\hline & Optimum temperature & $25-30^{\circ} \mathrm{C}$ & IDA \\
\hline & pH range; Optimum & $6-9 ; 8$ & IDA \\
\hline & Carbon source & D-glucose, lactose, acetate, bicarbonate & TAS [1] \\
\hline MIGS-6 & Habitat & Terrestrial, Black shale & TAS [1] \\
\hline MIGS-6.3 & Salinity & No growth with $>3.5 \% \mathrm{NaCl}(w / v)$ & IDA \\
\hline MIGS-22 & Oxygen requirement & Aerobic & TAS [1] \\
\hline MIGS-15 & Biotic relationship & free-living & TAS [1] \\
\hline MIGS-14 & Pathogenicity & Not reported & NAS \\
\hline MIGS-4 & Geographic location & Lockatong formation, New Jersey, USA & TAS [1] \\
\hline MIGS-5 & Sample collection & 2005 & IDA \\
\hline MIGS-4.1 & Latitude & 40.289329 & IDA \\
\hline MIGS-4.2 & Longitude & -74.814366 & IDA \\
\hline MIGS-4.4 & Altitude & $60 \mathrm{~m}$ & IDA \\
\hline
\end{tabular}

These evidence codes are from the Gene Ontology project [31]

${ }^{\mathrm{a}}$ Evidence codes

IDA Inferred from Direct Assay, TAS Traceable Author Statement (i.e., a direct report exists in the literature), NAS Non-traceable Author Statement (i.e., not directly observed for the living, isolated sample, but based on a generally accepted property for the species, or anecdotal evidence)

\section{Extended feature descriptions}

Bosea sp. WAO cells are Gram-negative, aerobic, motile, and rod shaped. Colonies on trypticase soy agar are smooth, mucoid, round, convex, and beige with a diameter as large as $10 \mathrm{~mm}$ after 2 weeks at $30{ }^{\circ} \mathrm{C}$. Colonies on minimal salts medium supplemented with $5 \mathrm{mM}$ sodium thiosulfate are smooth, round, white and only grow to a diameter of $2 \mathrm{~mm}$ after 2 weeks at $30{ }^{\circ} \mathrm{C}$. Optimal growth occurs at a temperature range from +25 to $30{ }^{\circ} \mathrm{C}$ and pH 6 to 9 with an optimum at $\mathrm{pH} 8$ (Table 1). Growth did not occur at salinity $>3.5 \% \mathrm{w} / \mathrm{v}$ of $\mathrm{NaCl}$. Cells will grow freely floating or attached to a mineral surface as shown in Fig. 8.

Strain WAO is a strict aerobe that can grow heterotrophically on acetate, glucose, and lactate in addition to autotrophically on carbon dioxide with the electron donors arsenite, thiosulfate, polysulfide, and elemental sulfur.
The organism is also able to grow on the mineral arsenopyrite (FeAsS) by oxidizing both the arsenic and sulfur to produce sulfate and arsenate. No growth was observed under aerobic conditions with the aromatic compounds phenol, benzoate or ferulic acid or with the electron donors sulfite, ammonium, nitrite, selenite, or chromium(III). This organism was enriched from pulverized black shale that contained high levels of arsenic. The initial enrichment cultures using the shale material were amended with $5 \mathrm{mM}$ arsenite and then serially diluted until purity was obtained [1].

\section{Genome sequencing information Genome project history}

Bosea sp. WAO was selected for sequencing based on the organism's ability to grow both heterotrophically and 
63 79

Bosea vaviloviae Vaf-18

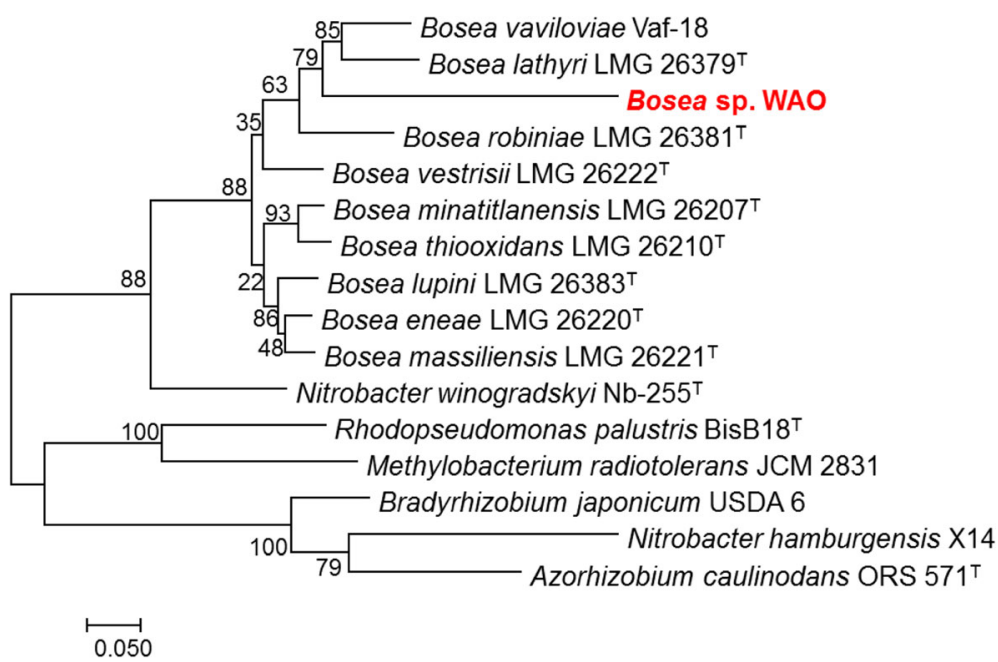

Fig. 2 Molecular Phylogenetic analysis by Maximum Likelihood method of aligned concatenated $a t p D$, dnaK, gyrB, recA, and rpoB. The evolutionary history was inferred by using the Maximum Likelihood method based on the Tamura-Nei model [1]. The tree with the highest log likelihood ( 13842.8588$)$ is shown. The percentage of trees in which the associated taxa clustered together is shown next to the branches. Initial tree(s) for the heuristic search were obtained automatically by applying Neighbor-Join and BioNJ algorithms to a matrix of pairwise distances estimated using the Maximum Composite Likelihood (MCL) approach, and then selecting the topology with superior log likelihood value. The tree is drawn to scale, with branch lengths measured in the number of substitutions per site. The analysis involved 16 nucleotide sequences. All positions containing gaps and missing data were eliminated. There were a total of 1413 positions in the final dataset. Evolutionary analyses were conducted in MEGA7 [21]

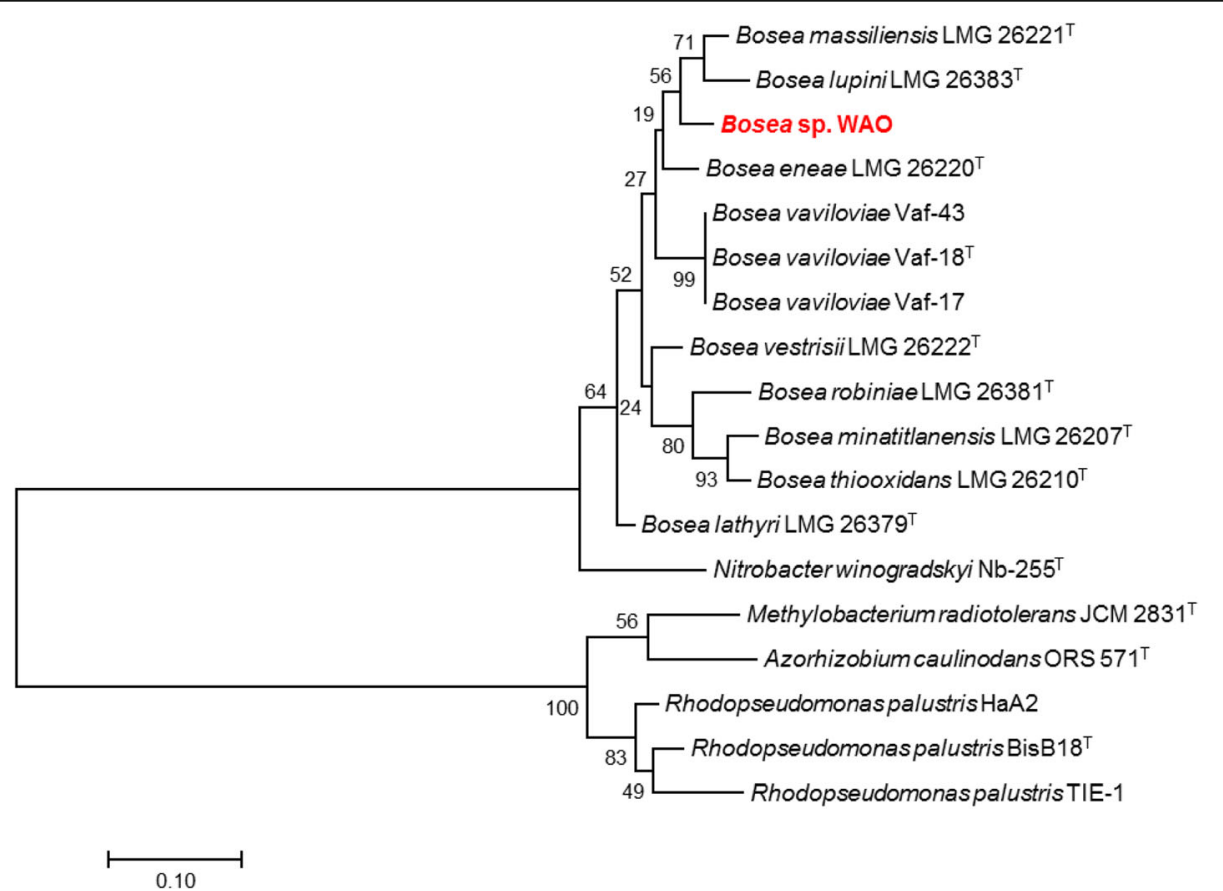

Fig. 3 Molecular Phylogenetic analysis by Maximum Likelihood method of the aptD gene. The evolutionary history was inferred by using the Maximum Likelihood method based on the Tamura-Nei model [19]. A Phylogenetic tree highlighting the position of Bosea sp. WAO relative to the other Bosea spp. and related organisms based on the aptD gene. The tree with the highest log likelihood (- 2412.0185) is shown. The percentage of trees in which the associated taxa clustered together is shown next to the branches. Initial tree(s) for the heuristic search were obtained automatically by applying Neighbor-Join and BioNJ algorithms to a matrix of pairwise distances estimated using the Maximum Composite Likelihood (MCL) approach, and then selecting the topology with superior log likelihood value. The tree is drawn to scale, with branch lengths measured in the number of substitutions per site. The analysis involved 18 nucleotide sequences. All positions containing gaps and missing data were eliminated. There were a total of 361 positions in the final dataset. Evolutionary analyses were conducted in MEGA7 [20]. Type strains are indicated with a superscript T 


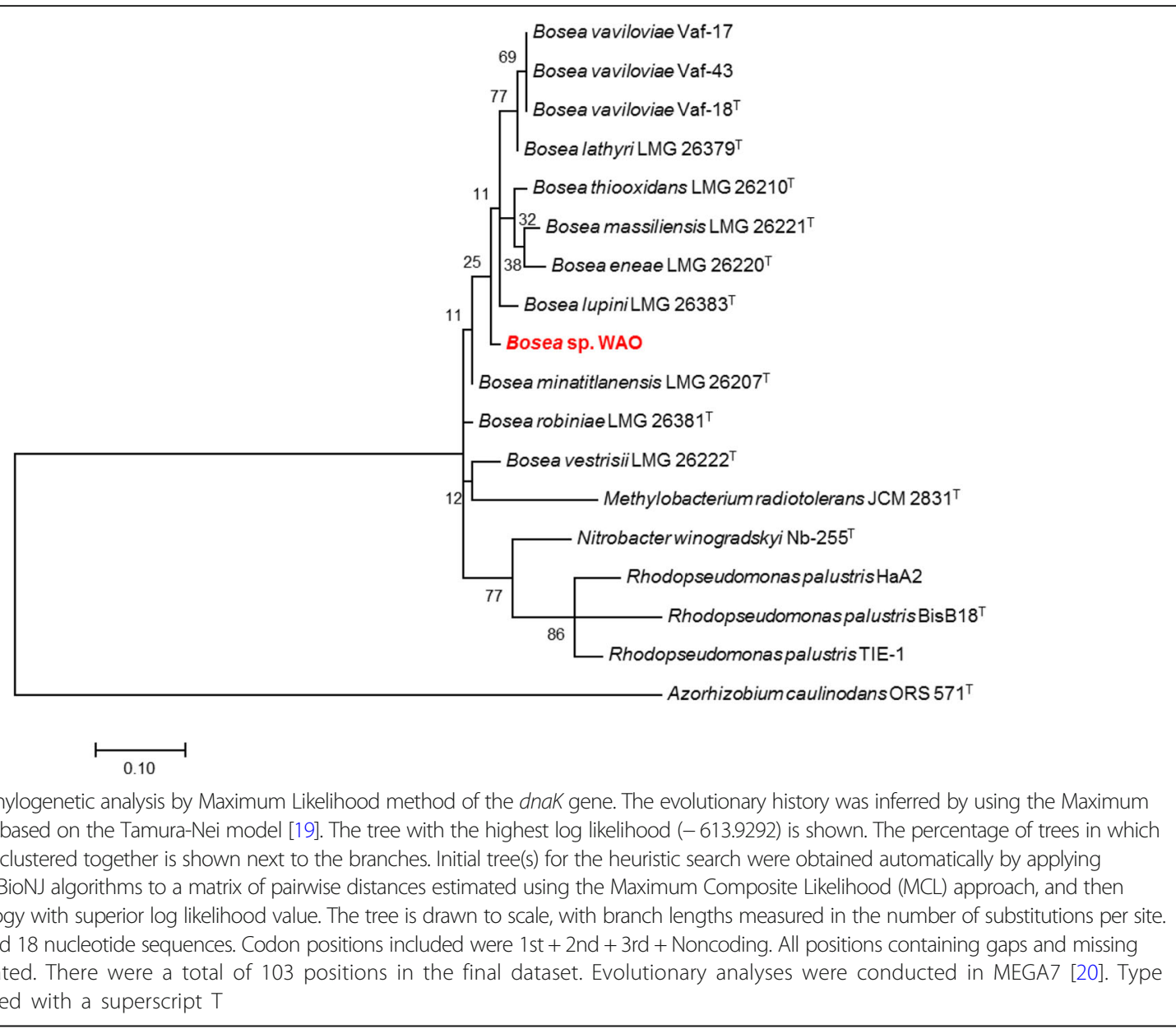

chemolithoautotrophically with arsenite and reduced sulfur compounds. Sequencing and assembly was completed at the Rutgers School of Environmental and Biological Sciences Genome Cooperative. A paired-end library was constructed using an Illumina Nextera Kit and sequenced using an Illumina Genome Analyzer IIX (Illumina Inc., San Diego, CA). The sequence assembly was performed using a CLC Genomics Workbench 5.1 (CLC Bio, Cambridge, MA). The draft genome was submitted to NCBI Whole Genome Shotgun (WGS) and to the JGI Integrated Microbial Genomes/ Expert Review (IMG/ER). A summary of the project is shown in Table 2.

\section{Growth conditions and genomic DNA preparation}

A culture of Bosea sp. WAO (GeneBank: DQ986321.1, DSM 102914) was grown in a dilute (50\% normal strength) trypticase soy broth amended with $5 \mathrm{mM}$ sodium arsenite and $5 \mathrm{mM}$ sodium thiosulfate then incubated at $30{ }^{\circ} \mathrm{C}$ on an orbital shaker for maximum oxygen exchange. Once turbid genomic DNA was extracted using the MoBio Powersoil Kit following manufacturer's directions with the modification that DNA was eluted into $100 \mathrm{uL}$ water instead of buffer.

\section{Genome sequencing and assembly}

A paired-end library was constructed using an Illumina Nextera Kit and sequenced using an Illumina Genome Analyzer IIX (Illumina Inc., San Diego, CA). The sequence assembly was performed using the CLC Genomics Workbench 5.1 (CLC Bio, Cambridge, MA). An average coverage of $240 \times$ and a mean read length of $106 \mathrm{bp}$ was obtained. The genome was assembled into 42 contigs with no additional gap closures.

\section{Genome annotation}

Genes were identified using the standard operating procedures of the DOE-JGI Microbial Genome Annotation pipeline [9] and The RAST Server: Rapid Annotation using subsystem technology [11, 12]. JGI-IMG/ER was used to obtain COG identities and overall statistics of 


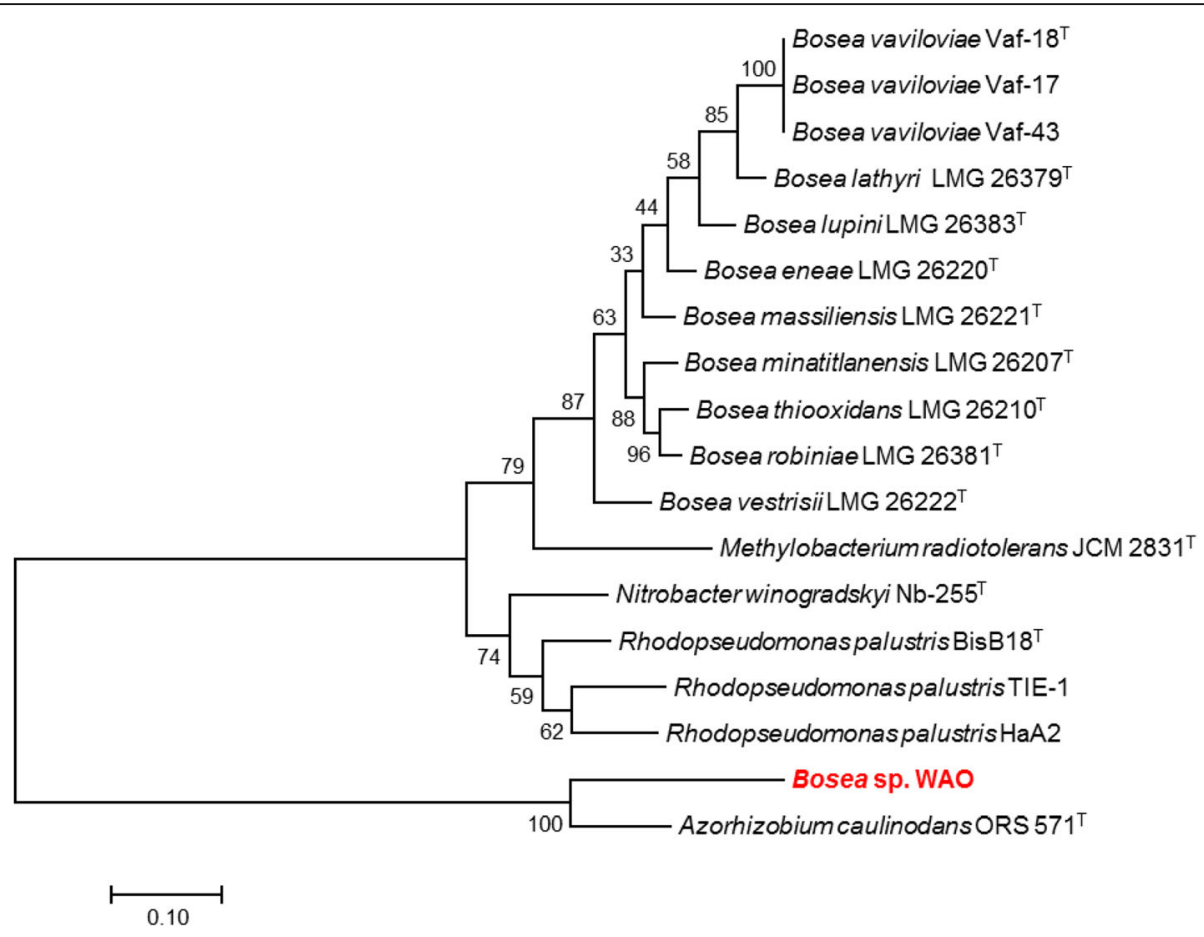

Fig. 5 Molecular Phylogenetic analysis by Maximum Likelihood method of gyrB gene. The evolutionary history was inferred by using the Maximum Likelihood method based on the Tamura-Nei model [19]. The tree with the highest log likelihood (-4279.1901) is shown. The percentage of trees in which the associated taxa clustered together is shown next to the branches. Initial tree(s) for the heuristic search were obtained automatically by applying Neighbor-Join and BioNJ algorithms to a matrix of pairwise distances estimated using the Maximum Composite Likelihood (MCL) approach, and then selecting the topology with superior log likelihood value. The tree is drawn to scale, with branch lengths measured in the number of substitutions per site. The analysis involved 18 nucleotide sequences. Codon positions included were 1st +2 nd $+3 r d+$ Noncoding. All positions containing gaps and missing data were eliminated. There were a total of 508 positions in the final dataset. Evolutionary analyses were conducted in MEGA7 [20]. Type strains are indicated with a superscript T

the genome. RAST was used to identify functional genes of interest involved in sulfur and arsenic metabolism.

\section{Genome properties}

The draft genome is $6,125,776$ bp with $66.84 \% \mathrm{G}+\mathrm{C}$ content. There are 62 RNA genes, 1 each of $5 \mathrm{~S}$ rRNA, $16 \mathrm{~S}$ rRNA, and 23S rRNA, and 46 tRNA, plus 13 unclassified RNA (Table 3). Of the predicted 5727 genes, 5665 or $98.92 \%$ are protein-coding genes, with $82.77 \%$ identified with protein function. The draft genome contains no identified pseudo genes. Of the protein-coding genes 4193 were sorted into COG functional categories. The COG categories are broken down in Table 4. COG analysis assigned a large number of genes to amino acid transport and metabolism (13.76\%), transcription (8. $13 \%)$, inorganic ion transport and metabolism (8.06\%), and energy production and conservation (6.97\%). Bosea sp. WAO has 53 genes encoding for cytochromes alone. RAST subsystem analysis placed $44 \%$ of the protein coding genes into subsystem categories with the largest percentage assigned to amino acids and derivatives. The genome sequence was deposited in GenBank ID JXTJ00000000.

\section{Extended insights}

Ten other genome sequences of Bosea spp. are publicly available of which four are validly named and characterized to the species level: $B$. thiooxidans CGMCC 9174 V5_1, B. lathyri DSM $26656^{\mathrm{T}}$, B. lupini DSM $26673^{\mathrm{T}}$, B. vaviloviae strain SD260 and six uncharacterized: Bosea sp. 117, Bosea sp. UNC402CLCol, Bosea sp. LC85, Bosea sp. OK403, Bosea sp. AAP35, and Bosea sp. AAP25. Only B. thiooxidans CGMCC 9174 V5_1 and B. vaviloviae strain SD260 are complete genomes. Table 5 details the basic characteristics of the ten genomes. The genomes range in size from $4.4 \mathrm{Mb}$ to $6.6 \mathrm{Mb}$ and $\mathrm{G}+\mathrm{C}$ content between 64 to $68 \%$, a predicated gene number range from 3984 to 6267 . Bosea $s p$. WAO's genome size (6.1 Mb), number of predicted genes (5727), number genes with function (4570), and number placed in COGs (4193) are all higher than the average for the draft genomes. However, both the percentage values for genes with functional predication (79.8\%) and percentage in 


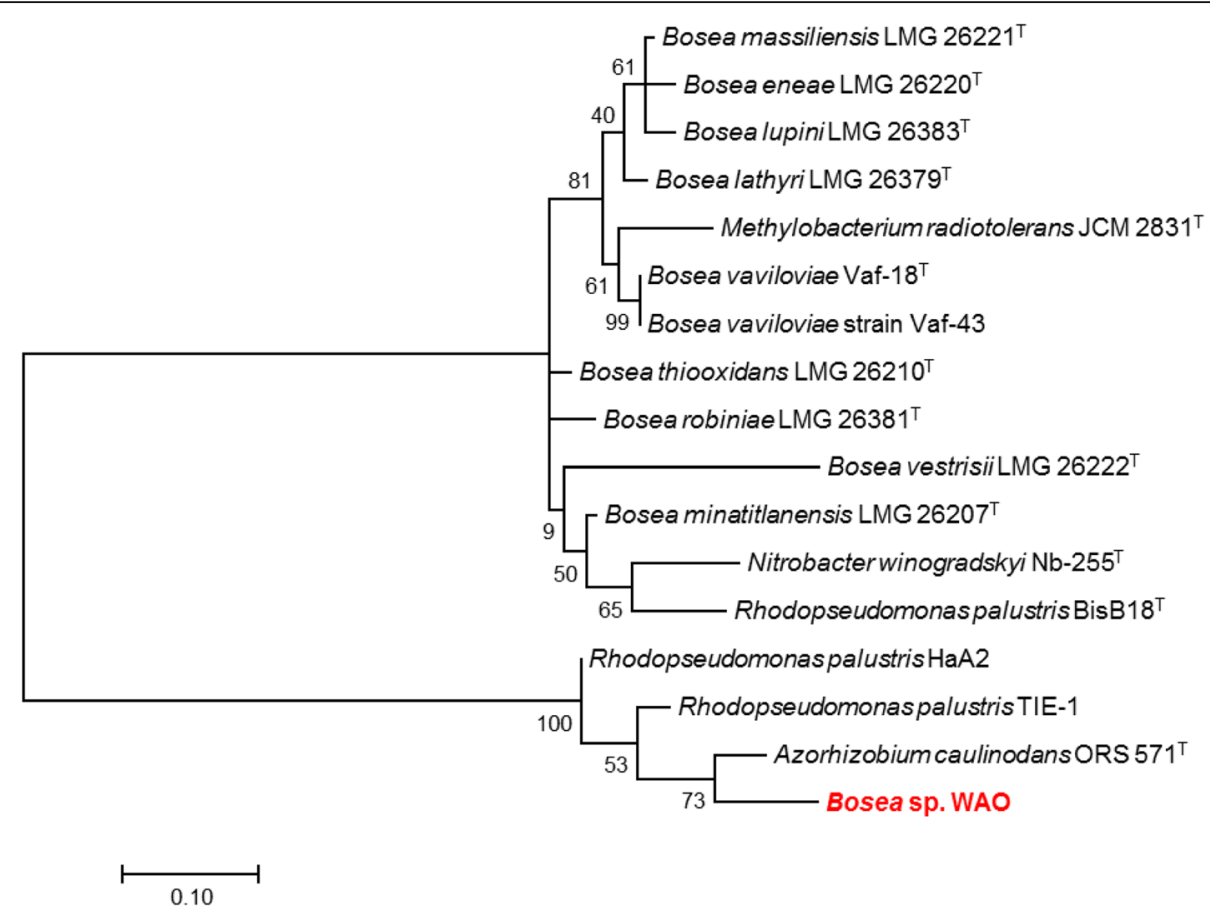

Fig. 6 Molecular Phylogenetic analysis by Maximum Likelihood method of recA gene. The evolutionary history was inferred by using the Maximum Likelihood method based on the Tamura-Nei model [19]. The tree with the highest log likelihood (-1263.1252) is shown. The percentage of trees in which the associated taxa clustered together is shown next to the branches. Initial tree(s) for the heuristic search were obtained automatically by applying Neighbor-Join and BioNJ algorithms to a matrix of pairwise distances estimated using the Maximum Composite Likelihood (MCL) approach, and then selecting the topology with superior log likelihood value. The tree is drawn to scale, with branch lengths measured in the number of substitutions per site. The analysis involved 17 nucleotide sequences. Codon positions included were 1st $+2 \mathrm{nd}+3 \mathrm{rd}+\mathrm{Noncoding}$. All positions containing gaps and missing data were eliminated. There were a total of 190 positions in the final dataset. Evolutionary analyses were conducted in MEGA7 [20]. Type strains are indicated with a superscript T

COGs (73.2\%) are similar to the average values for the draft genomes. B. thiooxidans CGMCC 9174 V5_1, B. vaviloviae strain SD260, Bosea sp. 117 and Bosea sp. UNC402CLCol contain pseudo genes. None of the IMG database genomes have been finished with scaffold numbers ranging between 16 and 72 .

\section{Arsenite oxidation}

Bosea sp. WAO is able to grow under chemolithoautotrophic conditions with arsenite in addition to growing under heterotrophic conditions. Metabolic studies indicated that the organism was able to stoichiometically oxidize the electron donors As(III) to As(V). Aerobic arsenite oxidation occurs using the aio genes renamed to reduce confusion from aso, aro and aox, which were formerly used to identify these genes in different organisms [13]. aio $A$ encodes for a large molybdopterin containing subunit with a guanosine dinucleotide at the active site and $a i o B$ encodes for a small Rieske subunit [13-15]. This pathway has a two component regulatory system that includes a sensor histidine kinase encoded by aios (aoxS, aroS) and a transcriptional regulator encoded by aioR (aoxR, aroR) [13-15]. For the initial publication of Bosea sp. WAO, only the large subunit gene for the arsenite oxidation pathway aioA (EF015463) was amplified by traditional PCR $[1,16]$. Analysis of the genome herein revealed that the arsenite oxidation pathway was complete with Bosea sp. WAO possessing both the small subunit $a i o B$ and reconfirming the large subunit aio $A$ in addition to the remaining genes in the pathway. Of the available genomes only Bosea sp. WAO, and Bosea sp. 117 genomes contain both the large and small arsenite subunits with an amino acid similarity of $78 \%$ for AioA and $73 \%$ for AioB. The genes within the arsenite oxidation operon are in the same order (Fig. 9). The operon begins with a sensor histidine kinase, aioS, followed by a transcriptional response regulator, aioR, and then aioB, followed by aio $A$.

\section{Reduced sulfur compound oxidation}

Bosea sp. WAO is also able to grow under chemolithoautotrophic conditions with thiosulfate, polysulfide, and elemental sulfur. Metabolic studies indicated that the organism is able to stoichiometically oxidize the 


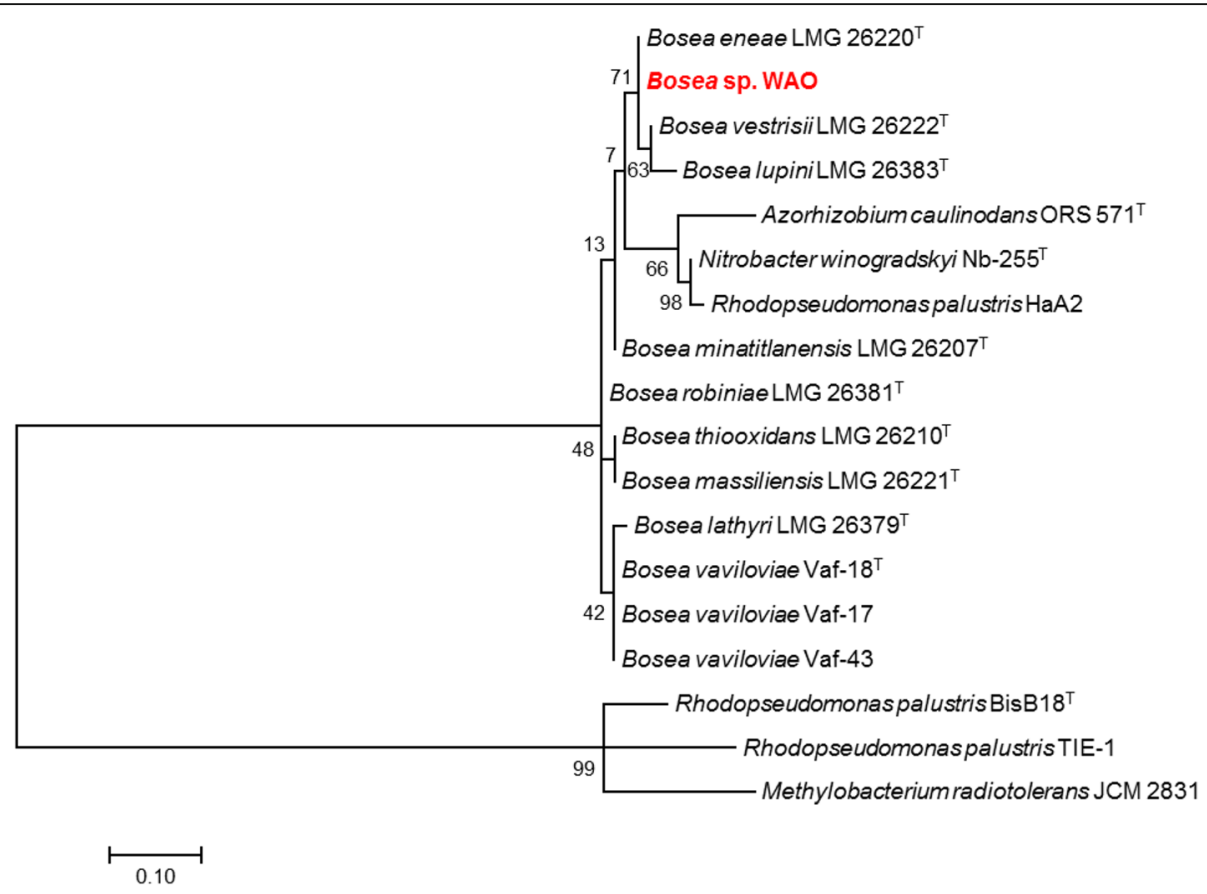

Fig. 7 Molecular Phylogenetic analysis by Maximum Likelihood method of rpoB gene. The evolutionary history was inferred by using the Maximum Likelihood method based on the Tamura-Nei model [19]. The tree with the highest log likelihood (-419.8311) is shown. The percentage of trees in which the associated taxa clustered together is shown next to the branches. Initial tree(s) for the heuristic search were obtained automatically by applying Neighbor-Join and BioNJ algorithms to a matrix of pairwise distances estimated using the Maximum Composite Likelihood (MCL) approach, and then selecting the topology with superior log likelihood value. The tree is drawn to scale, with branch lengths measured in the number of

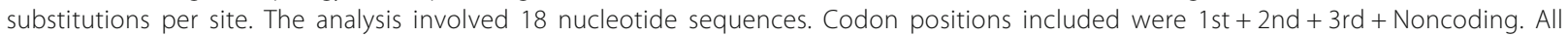
positions containing gaps and missing data were eliminated. There were a total of 76 positions in the final dataset. Evolutionary analyses were conducted in MEGA7 [20]. Type strains are indicated with a superscript T

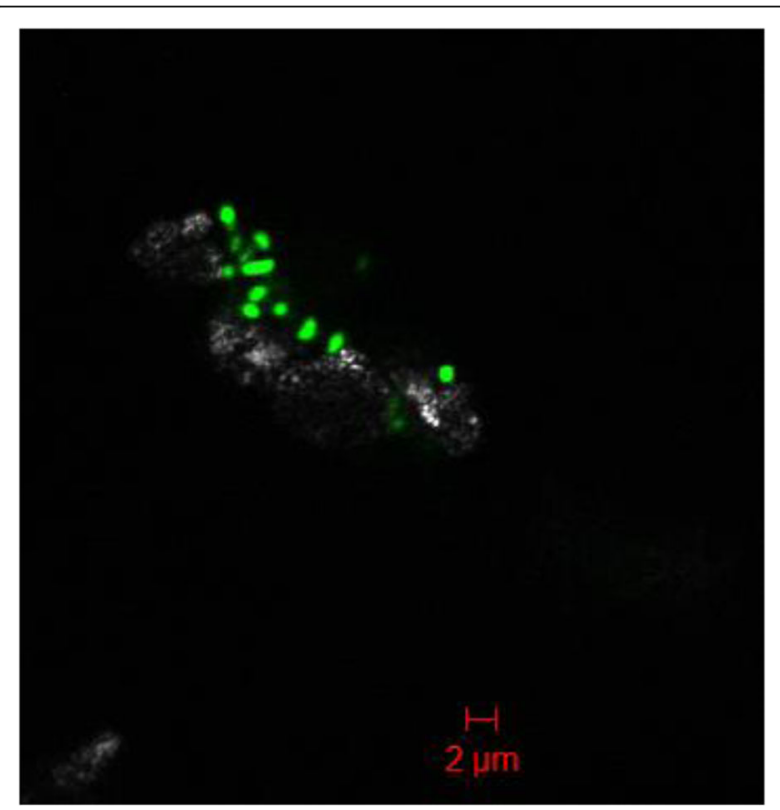

Fig. 8 Confocal microscopy of Bosea sp. WAO. Bosea sp. WAO (green) was stained with DAPI and imaged growing on the surface of a cadmium sulfide particle (faint white/grey) in a mostly black background
Table 2 Project information

\begin{tabular}{lll}
\hline MIGS ID & Property & Term \\
\hline MIGS 31 & Finishing quality & Draft \\
MIGS-28 & Libraries used & One pair-end \\
MIGS 29 & Sequencing platforms & Illumina Genome Analyzer IIX \\
MIGS 31.2 & Fold coverage & 240x \\
MIGS 30 & Assemblers & CLC Genomics Workbench 5.1 \\
MIGS 32 & Gene calling method & Glimmer \\
& Locus Tag & DK26 \\
& Genbank ID & JXTJ00000000 \\
& GenBank Date of Release & January 8, 2016 \\
& GOLD ID & Gp0113237 \\
& BIOPROJECT & PRJNA243637 \\
MIGS 13 & Source Material Identifier & DSM 102914 \\
& Project relevance & Environmental, biogeochemical \\
& & cycling of arsenic and sulfur \\
\hline
\end{tabular}


Table 3 Genome statistics

\begin{tabular}{lll}
\hline Attribute & Value & \% of total \\
\hline Genome size (bp) & $6,125,776$ & 100.00 \\
DNA coding (bp) & $5,469,601$ & 89.29 \\
DNA G + C (bp) & $4,094,621$ & 66.84 \\
DNA scaffolds & 42 & 100.00 \\
Total genes & 5727 & 100.00 \\
Protein coding genes & 5665 & 98.92 \\
RNA genes & 62 & 1.08 \\
Pseudo genes & 0 & 0 \\
Genes in internal clusters & & \\
Genes with function prediction & 4740 & 82.77 \\
Genes assigned to COGs & 4193 & 73.21 \\
Genes with Pfam domains & 4837 & 84.46 \\
Genes with signal peptides & 635 & 11.09 \\
Genes with transmembrane helices & 1391 & 24.29 \\
CRISPR repeats & 0 & 0 \\
\hline
\end{tabular}

electron donor $\mathrm{S}_{2} \mathrm{O}_{3}$ to $\mathrm{SO}_{4}{ }^{2-}$. The sox gene cluster is a pathway consisting of seven essential genes, sox $X Y Z A B C D$, that code for proteins required for direct oxidation from sulfide to sulfate in vivo [17]. The genome analysis indicated that strain WAO possesses all the genes necessary for the sulfur oxidation pathway. KEGG analysis indicated genes are all present to code for the enzymes SoxB, SoxX, SoxY, SoxA, SoxC, and SoxD to allow for complete oxidation of $\mathrm{S}_{2} \mathrm{O}_{3}$ to $\mathrm{SO}_{4}{ }^{2-}$. Bosea sp. WAO, in addition to $B$. thiooxidans CGMCC 9174 V5_1, Bosea sp. 117, Bosea sp. LC85, and B. lupini contain the complete sox system. For the four genomes available in IMG the overall gene order in the operons are the same for all organisms; however, Bosea sp. WAO and $B$. lupini have $\operatorname{sox} A$ and $\operatorname{sox} X$ on the plus strand and soxY, soxZ, soxB, soxC, soxD on the minus strand (Fig. 10). While Bosea sp. 117 and Bosea sp. LC85 have the genes on the reverse strands with $\operatorname{sox} Y, \operatorname{sox} Z$, $\operatorname{sox} B, \operatorname{sox} C$, $\operatorname{sox} D$ on the plus and soxA and $\operatorname{sox} X$ on the minus strand (Fig. 10). Comparison of the translated nucleotide sequence of $s o x B$ from Bosea sp. WAO to the translated $\operatorname{sox} B$ of the other five organisms showed that the protein sequence is $90 \%$ similar to Bosea sp. LC $85,88 \%$

Table 4 Number of genes associated with general COG functional categories

\begin{tabular}{|c|c|c|c|}
\hline Code & Value & \%age & Description \\
\hline J & 212 & 4.44 & Translation, ribosomal structure and biogenesis \\
\hline A & 0 & 0 & RNA processing and modification \\
\hline K & 388 & 8.13 & Transcription \\
\hline L & 112 & 2.35 & Replication, recombination and repair \\
\hline B & 3 & 0.06 & Chromatin structure and dynamics \\
\hline $\mathrm{D}$ & 29 & 0.61 & Cell cycle control, cell division, chromosome partitioning \\
\hline V & 109 & 2.28 & Defense mechanisms \\
\hline $\mathrm{T}$ & 213 & 4.46 & Signal transduction mechanisms \\
\hline M & 239 & 5.01 & Cell wall/membrane biogenesis \\
\hline N & 75 & 1.57 & Cell motility \\
\hline$U$ & 60 & 1.26 & Intracellular trafficking and secretion \\
\hline $\mathrm{O}$ & 175 & 3.66 & Posttranslational modification, protein turnover, chaperones \\
\hline C & 333 & 6.97 & Energy production and conversion \\
\hline G & 276 & 5.78 & Carbohydrate transport and metabolism \\
\hline E & 657 & 13.76 & Amino acid transport and metabolism \\
\hline $\mathrm{F}$ & 102 & 2.14 & Nucleotide transport and metabolism \\
\hline $\mathrm{H}$ & 233 & 4.88 & Coenzyme transport and metabolism \\
\hline I & 249 & 5.21 & Lipid transport and metabolism \\
\hline P & 385 & 8.06 & Inorganic ion transport and metabolism \\
\hline Q & 155 & 3.25 & Secondary metabolites biosynthesis, transport and catabolism \\
\hline $\mathrm{R}$ & 470 & 9.84 & General function prediction only \\
\hline S & 268 & 5.61 & Function unknown \\
\hline- & 1534 & 26.79 & Not in COGs \\
\hline
\end{tabular}

The total is based on the total number of protein coding genes in the genome 
Table 5 Comparison of basic genome features of Bosea spp.

\begin{tabular}{|c|c|c|c|c|c|c|c|c|c|}
\hline $\begin{array}{l}\text { Genome } \\
\text { Name }\end{array}$ & Status $^{\mathrm{a}}$ & $\begin{array}{l}\text { Genome Size } \\
\text { (Mbp) }\end{array}$ & $\begin{array}{l}\mathrm{G}+\mathrm{C} \\
\text { Content (\%) }\end{array}$ & $\begin{array}{l}\text { Gene } \\
\text { Count }\end{array}$ & $\begin{array}{l}\text { No. of protein coding genes } \\
\text { w/ function prediction }\end{array}$ & $\begin{array}{l}\text { Percentage } \\
(\%)\end{array}$ & $\begin{array}{l}\text { No. of protein coding } \\
\text { genes in COGs }\end{array}$ & $\begin{array}{l}\text { Percentage } \\
\text { (\%) }\end{array}$ & $\begin{array}{l}\text { IMG Taxon } \\
\text { ID }\end{array}$ \\
\hline $\begin{array}{l}\text { Bosea sp. } \\
\text { WAO [1] }\end{array}$ & $\mathrm{D}$ & 6.12 & 67 & 5727 & 4570 & 79.8 & 4193 & 73.2 & 2615840542 \\
\hline $\begin{array}{l}\text { Bosea sp. } \\
\text { LC85 [32] }\end{array}$ & PD & 6.56 & 65 & 6267 & 4975 & 79.4 & 4548 & 72.6 & 2609460206 \\
\hline $\begin{array}{l}\text { Bosea sp. } \\
\text { UNC402CLCol }\end{array}$ & PD & 5.61 & 67 & 5389 & 4375 & 81.1 & 4067 & 75.5 & 2579779168 \\
\hline $\begin{array}{l}\text { Bosea lupini } \\
\text { DSM } 26673[6]\end{array}$ & $\mathrm{D}$ & 6.13 & 67 & 5985 & 4752 & 79.4 & 4396 & 73.4 & 2634166302 \\
\hline Bosea sp. OK403 & $\mathrm{D}$ & 6.64 & 65 & 6099 & 5066 & 83.1 & 4396 & 77.0 & 2609459641 \\
\hline Bosea sp. AAP25 & PD & 4.14 & 64 & 3984 & 3023 & 75.9 & 2651 & 66.5 & 2636415410 \\
\hline $\begin{array}{l}\text { Bosea lathyri } \\
\text { DSM } 26656 \text { [21] }\end{array}$ & D & 5.91 & 65 & 5559 & 4476 & 80.5 & 4120 & 74.1 & 2622736433 \\
\hline Bosea sp. 117 & PD & 4.63 & 68 & 4344 & 3639 & 83.8 & 3366 & 77.5 & 2562617052 \\
\hline Bosea sp. AAP35 & PD & 4.46 & 66 & 4298 & 3435 & 79.9 & 3144 & 73.1 & 2636415883 \\
\hline $\begin{array}{l}\text { Bosea vaviloviae } \\
\text { strain SD260 }\end{array}$ & $\mathrm{F}$ & 5.60 & 66 & 5487 & 4260 & 77.6 & 3839 & 69.9 & 2654587694 \\
\hline $\begin{array}{l}\text { Bosea thiooxidans } \\
\text { CGMCC } 9174 \text { V5_1 }\end{array}$ & $\mathrm{F}$ & 5.46 & 67 & 5176 & - & - & - & - & N/A \\
\hline
\end{tabular}

These data were obtained from the IMG/ER platform [9] and NCBI genomes

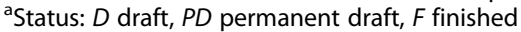

similar to B. lupini and B. thiooxidans CGMCC 9174 V5_1, and 70\% similar to Bosea sp. 117. The presence of all the genes in the same order suggests other strains in addition to the experimentally confirmed Bosea thiooxidans $\mathrm{BI}-42^{\mathrm{T}}$, may be able to perform thiosulfate oxidation.

\section{Additional metabolic pathways}

The Calvin Cycle consists of 13 enzymatic reactions with the enzyme ribulose-1,5 bisphosphate carboxylase/oxygenase ( $\mathrm{RuBisCO})$ responsible for the carbon fixation step [18]. For the initial publication of Bosea sp. WAO the type II ribulose-1,5'bisphosphate carboxylase/oxygenase (RuBisCO) was amplified by traditional PCR [1, 16]. Analysis for the remaining genes of the CalvinBenson-Bassham Cycle for carbon fixation indicated that all the other required genes were present for carbon fixation to occur. Nine of the available genomes have a match for strain WAO's ribulose 1,5-bisphosphate carboxylase amino acid sequence: $B$. thiooxidans

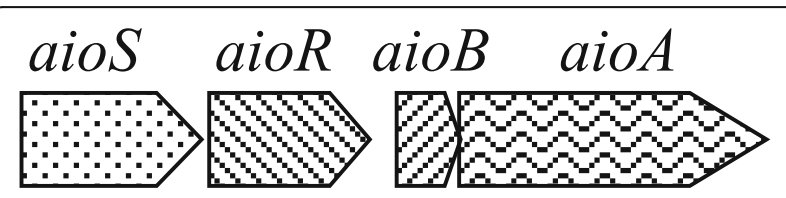

Fig. 9 Operon structure for arsenite oxidation viewed 5'-3' direction on the plus strand. The gene order is the same in both Bosea sp. WAO and Bosea sp. 117 with a senor histidine kinase, aioS, then a transcriptional response regulator, aio $R$, followed by the $a i o B$ and aioA genes
CGMCC 9174 V5_1, (85\%), B. lathyri DSM 26656 ${ }^{\mathrm{T}}$, (86\%), B. lupini DSM 26673 ${ }^{\mathrm{T}},(82 \%)$, B. vaviloviae strain SD260, (85\%), Bosea sp. 117, (72\%), Bosea sp. UNC402CLCol, (85\%), Bosea sp. LC85, (84\%), Bosea sp. OK403, (87\%), and Bosea sp. AAP35, (84\%). Since RuBisCO is considered a biomarker for the Calvin Cycle this suggests carbon fixation maybe be widespread in this genus despite the limited experimental evidences.

Additional KEGG analysis indicated incomplete pathways for nitrogen reduction. Bosea sp. WAO possesses some genes for each of the reductive pathways but each is incomplete supporting the observation that no growth occurred when nitrate was provided as an electron acceptor. No genes involved in ammonia oxidation were identified again supporting the absence of growth when cultivated under those conditions [1]. Using IMG/ER Pipeline analysis Bosea sp. WAO was determined to be prototrophic for L-aspartate, L-glutamate, and glycine; auxotrophic for L-lysine, L-alanine, L-phenylalanine, Ltyrosine, L-tryptophan, L-histine, L-arginine, L-isoleucine, L-leucine, and L-valine; and not able to synthesize selenocycteine synthesizer or biotin based on the draft of the genome [9]. Using the SEED viewer Bosea sp. WAO has complete pathways for the: tricarboxylic acid cycle, pentose phosphate pathway, acetyl-coA acetogenesis pathway, methylglyoxal metabolism, dihydroxyacetone kinases, catechol branch of beta-ketoadipate pathway, glycerol and clycerol-3-phosphate uptake and utilization, D-ribose utilization, deoxyribose and deoxynucleoside catabolism, and lactate utilization. 


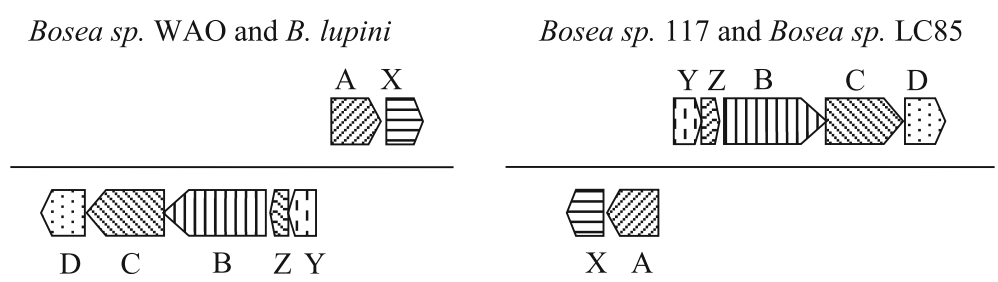

Fig. 10 Operon structure for the sox genes for thiosulfate oxidation. The orientation is $5^{\prime}-3^{\prime}$ with the plus strand on top. The orientation of the genes for Bosea sp. WAO and Bosea lupini are the same while Bosea sp. LC85 and Bosea sp. 117 have the same orientation. These operons are inverted between the plus and minus strands

\section{Conclusions}

Bosea sp. WAO is able to grow chemolithoautotrophically on both arsenite and reduced sulfur compounds. It was originally enriched from pyritic shale obtained from a rock outcropping containing arsenic in the Lockatong geological formation in the Newark Basin near Trenton, New Jersey [1]. The draft genome is 6.1 Mbps and a G + C content of $66.84 \%$. COG analysis for Bosea sp. WAO assigned a large number of genes to amino acid transport and metabolism (13.76\%), transcription (8.13\%), inorganic ion transport and metabolism $(8.06 \%)$, and energy production and conservation (6.97\%). Bosea sp. WAO has 53 genes encoding for cytochromes alone. Strain WAO is able to engage in the oxidative part of biogeochemical cycling and grow autotrophically when nutrient conditions are low. When conditions favor heterotrophic growth, however, the organism is able to rapidly increase in biomass and maintain its population under the varying conditions that expected to prevail at an oxic mineral surface.

\section{Acknowledgements}

Technical support was provided by the Rutgers School of Environmental and Biological Sciences Genome Cooperative.

\section{Funding}

This work was supported in part by the New Jersey Water Resources Research Institute at Rutgers University.

\section{Authors' contributions}

ABW performed the laboratory experiments, analysed the assembled genome sequence data and wrote the draft manuscript. NY and LYY participated in the design of the study and revised the manuscript. All authors read and approved the final manuscript.

\section{Competing interests}

The authors declare that they have no competing interests.

\section{Publisher's Note}

Springer Nature remains neutral with regard to jurisdictional claims in published maps and institutional affiliations.

\section{Author details}

'Division of Life Sciences, Rutgers University, The State University of New Jersey, Piscataway, New Jersey, USA. ${ }^{2}$ Department of Environmental Sciences, Rutgers University, The State University of New Jersey, New Brunswick, New Jersey, USA.
Received: 19 July 2017 Accepted: 21 March 2018

Published online: 10 April 2018

\section{References}

1. Rhine ED, Onesios KM, Serfes ME, Reinfelder JR, Young LY. Arsenic transformation and mobilization from minerals by the arsenite oxidizing strain WAO. Environ Sci Technol. 2008;42:1423-9.

2. de Souza JAM, Carrareto Alves LM, de Mello Varani A, de Macedo Lemos E. The family Bradyrhizobiaceae. In: Rosenberg E, DeLong EF, Lory S, Stackebrandt E, Thompson F, editors. The prokaryotes. Berlin Heidelberg: Springer; 2014. p. 135-54.

3. Das SK, Mishra AK, Tindall B, Rainey FA. Oxidation of thiosulfate by a new bacterium, Bosea thiooxidans. (strain Bl-42) gen. nov., sp. nov.: analysis of phylogeny based on chemotaxonomy and 165 ribosomal DNA sequencing. Int J Syst Bacteriol. 1996;46:981-7.

4. La Scola B, Mallet M, Grimont PAD, Raoult D. Bosea eneae sp. nov., Bosea massiliensis sp. nov. and Bosea vestrisii sp. nov., isolated from hospital water supplies, and emendation of the genus Bosea (Das et al. 1996). Int J Syst Evol Microbiol. 2003;53:15-20.

5. Ouattara AS, Assih EA, Thierry S, Cayol J, Labat M, Monroy O, et al. Bosea minatitlanensis sp. nov., a strictly aerobic bacterium isolated from an anaerobic digester. Int J Syst Evol Microbiol. 2003;53:1247-51.

6. De Meyer SE, Willems A. Multilocus sequence analysis of Bosea species and description of Bosea lupini sp. nov., Bosea lathyri sp. nov. and Bosea robiniae sp. nov., isolated from legumes. Int J Syst Evol Microbiol. 2012;62:2505-10.

7. Safronova VI, Kuznetsova IG, Sazanova AL, Kimeklis AK, Belimov AA, Andronov EE, et al. Bosea vaviloviae sp. nov., a new species of slow-growing rhizobia isolated from nodules of the relict species Vavilovia formosa (Stev.) Fed. Antonie Van Leeuwenhoek J Microb. 2015;107:911-20.

8. Kim OS, Cho YJ, Lee K, Yoon SH, Kim M, Na H, et al. Introducing EzTaxon-e: a prokaryotic $16 \mathrm{~S}$ rRNA gene sequence database with phylotypes that represent uncultured species. Int I Syst Evol Microbiol. 2012;62:716-21.

9. Markowitz VM, Chen IA, Palaniappan K, Chu K, Szeto E, Grechkin Y, et al. IMG: the integrated microbial genomes database and comparative analysis system. Nucleic Acids Res. 2012;40:D115-22.

10. Kim M, Oh H, Park S, Chun J. Towards a taxonomic coherence between average nucleotide identity and 16S rRNA gene sequence similarity for species demarcation of prokaryotes. Int J Syst Evol Microbiol. 2014;64:346-51.

11. Overbeek R, Begley T, Butler RM, Choudhuri JV, Chuang H, Cohoon M, et al. The subsystems approach to genome annotation and its use in the project to annotate 1000 genomes. Nucleic Acids Res. 2005;33:5691-702.

12. Aziz R, Bartels D, Best A, DeJongh M, Disz T, Edwards R, et al. The RAST server: rapid annotations using subsystems technology. BMC Genomics. 2008;9:75

13. Lett $M$, Muller $D$, Lièvremont $D$, Silver $S$, Santini J. Unified nomenclature for genes involved in prokaryotic aerobic arsenite oxidation. J Bacteriol. 2012; 194:207-8.

14. Silver S, Phung LT. Genes and enzymes involved in bacterial oxidation and reduction of inorganic arsenic. Appl Environ Microbiol. 2005;71:599-608.

15. Amend JP, Saltikov C, Lu G, Hernandez J. Microbial arsenic metabolism and reaction energetics. Rev Mineral Geochem. 2014;79:391-433.

16. Rhine ED, Ní Chadhain SM, Zylstra GJ, Young LY. The arsenite oxidase genes (aroAB) in novel chemoautotrophic arsenite oxidizers. Biochem Biophys Res Commun. 2007:354:662-7.

17. Friedrich CG, Rother D, Bardischewsky F, Quentmeier A, Fischer J. Oxidation of reduced inorganic sulfur compounds by bacteria: emergence of a common mechanism? Appl Environ Microbiol. 2001;67:2873-82. 
18. Shively JM, van Keulen G, Meijer WG. Something from almost nothing: carbon dioxide fixation in chemoautotrophs. Annu Rev Microbiol. 1998;52:191-230.

19. Tamura K, Nei M. Estimation of the number of nucleotide substitutions in the control region of mitochondrial DNA in humans and chimpanzees. Mol Biol Evol. 1993;10:512-26.

20. Kumar S, Stecher G, Tamura K. MEGA7: Molecular Evolutionary Genetics Analysis version 7.0 for bigger datasets. 2016.

21. Meyer B, Imhoff JF, Kuever J. Molecular analysis of the distribution and phylogeny of the soxB gene among sulfur-oxidizing bacteria - evolution of the sox sulfur oxidation enzyme system. Environ Microbiol. 2007;9:2957-77.

22. Field D, Garrity G, Gray T, Morrison N, Selengut J, Sterk P, et al. The minimum information about a genome sequence (MIGS) specification. Nat Biotechnol. 2008;26:541-7.

23. Woese CR, Kandler O, Wheelis ML. Towards a natural system of organisms: proposal for the domains archaea, bacteria, and eucarya. Proc Natl Acad Sci. 1990:87:4576-9.

24. Wang Q, Garrity GM, Tiedje JM, Cole JR. Naiive Bayesian classifier for rapid assignment of rRNA sequences into the new bacterial taxonomy. Appl Environ Microbiol. 2007;73:5261-7.

25. Garrity GM, Bell J, Lilburn T. Phylum XIV. Proteobacteria phyl. nov. In: Bergey's manual of systematic bacteriology, vol. Part B; 2005. p. 1.

26. Garrity GM, Bell JA, Lilburn T. Class I. Alphaproteobacteria class. nov. In: Garrity G, Brenner DJ, Krieg NR, Staley JT, editors. Bergey's manual ${ }^{\star}$ of systematic bacteriology. US: Springer; 2005. p. 1-574.

27. List Editor. Validation List No. 107. List of new names and new combinations previously effectively, but not validly, published, vol. 56; 2006. p. 1-6.

28. Kuykendall LD. Order Vi. Rhizobiales ord. nov. In: Garrity G, Brenner DJ, Krieg NR, Staley JT, editors. Bergey's manual ${ }^{\oplus}$ of systematic bacteriology. US: Springer; 2005. p. 324.

29. Garrity GM, Bell JA, Lilburn T. Family VII. Bradyrhizobiaceae fam. nov. In: Garrity G, Brenner DJ, Krieg NR, Staley JT, editors. Bergey's manual ${ }^{\bullet}$ of systematic bacteriology. US: Springer; 2005. p. 438.

30. Das SK. Genus V. Bosea Das, Mishra, TIndall, Rainey and Stackebrandt 1996, $985^{V P}$. In: Garrity G, Brenner DJ, Krieg NR, Staley JT, editors. Bergey's manual ${ }^{\circ}$ of systematic bacteriology. US: Springer; 2005. p. 459-461.

31. Gene Ontology Consortium. The Gene Ontology (GO) database and informatics resource. Nucleic Acids Res. 2004:32:D258-61.

32. Gan HY, Gan HM, Tarasco AM, Busairi NI, Barton HA, Hudson AO, et al. Whole-genome sequences of five oligotrophic bacteria isolated from deep within Lechuguilla Cave, New Mexico. Genome Announc. 2014;2 https://doi. org/10.1128/genomeA.01133-14.

\section{Submit your next manuscript to BioMed Central and we will help you at every step:}

- We accept pre-submission inquiries

- Our selector tool helps you to find the most relevant journal

- We provide round the clock customer support

- Convenient online submission

- Thorough peer review

- Inclusion in PubMed and all major indexing services

- Maximum visibility for your research

Submit your manuscript at www.biomedcentral.com/submit

CBiomed Central 